\title{
Inhaltsübersicht
}

\author{
Insolvenzordnung \\ Sechster Teil \\ Insolvenzplan \\ Erster Abschnitt \\ Aufstellung des Plans
}

$\begin{array}{ll}\mathbb{S} 217 & \text { Grundsatz } \\ \mathbb{\$} 218 & \text { Vorlage des Insolvenzplans } \\ \mathbb{S} 219 & \text { Gliederung des Plans } \\ \mathbb{S} 220 & \text { Darstellender Teil } \\ \mathbb{\$} 221 & \text { Gestaltender Teil } \\ \mathbb{S} 222 & \text { Bildung von Gruppen } \\ \mathbb{S} 223 & \text { Rechte der Absonderungsberechtigten } \\ \mathbb{S} 224 & \text { Rechte der Insolvenzgläubiger } \\ \mathbb{S} 225 & \text { Rechte der nachrangigen Insolvenzgläubiger } \\ \mathbb{S} 225 \mathrm{a} & \text { Rechte der Anteilsinhaber } \\ \mathbb{S} 226 & \text { Gleichbehandlung der Beteiligten } \\ \mathbb{S} 227 & \text { Haftung des Schuldners } \\ \mathbb{S} 228 & \text { Änderung sachenrechtlicher Verhältnisse } \\ \mathbb{S} 229 & \text { Vermögensübersicht. Ergebnis- und Finanzplan } \\ \mathbb{\$} 230 & \text { Weitere Anlagen } \\ \mathbb{\$} 231 & \text { Zurückweisung des Plans } \\ \mathbb{S} 232 & \text { Stellungnahmen zum Plan } \\ \mathbb{\$} 233 & \text { Aussetzung von Verwertung und Verteilung } \\ \mathbb{S} 234 & \text { Niederlegung des Plans }\end{array}$

Zweiter Abschnitt

Annahme und Bestätigung des Plans
Bearbeiter Joachim Münch Joachim Münch Joachim Münch Joachim Münch Joachim Münch Joachim Münch Joachim Münch Joachim Münch Joachim Münch Joachim Münch Joachim Münch Joachim Münch Joachim Münch Joachim Münch Joachim Münch Joachim Münch Joachim Münch Joachim Münch Joachim Münch

$\begin{array}{ll}\mathbb{2} 235 & \text { Erörterungs- und Abstimmungstermin } \\ \mathbb{S} 236 & \text { Verbindung mit dem Prüfungstermin } \\ \mathbb{S} 237 & \text { Stimmrecht der Insolvenzgläubiger } \\ \mathbb{S} 238 & \text { Stimmrecht der absonderungsberechtigten Gläubiger } \\ \mathbb{S} 238 \mathrm{a} & \text { Stimmrecht der Anteilsinhaber } \\ \mathbb{\$} 239 & \text { Stimmliste } \\ \mathbb{S} 240 & \text { Änderung des Plans } \\ \mathbb{S} 241 & \text { Gesonderter Abstimmungstermin } \\ \mathbb{\$} 242 & \text { Schriftliche Abstimmung } \\ \mathbb{S} 243 & \text { Abstimmung in Gruppen } \\ \mathbb{S} 244 & \text { Erforderliche Mehrheiten } \\ \mathbb{\$} 245 & \text { Obstruktionsverbot } \\ \mathbb{S} 246 & \text { Zustimmung nachrangiger Insolvenzgläubiger }\end{array}$

Christoph Kern Christoph Kern Christoph Kern Christoph Kern Christoph Kern Christoph Kern Christoph Kern Christoph Kern Christoph Kern Christoph Kern Christoph Kern Christoph Kern Christoph Kern 
Inhaltsübersicht

\begin{tabular}{|c|c|}
\hline $246 a$ & Zustimmung der Anteilsinhaber \\
\hline 247 & Zustimmung des Schuldners \\
\hline & Gerichtliche Bestätigung \\
\hline $48 \mathrm{a}$ & Gerichtliche Bestätigung einer Planberichtigung \\
\hline 249 & Bedingter Plan \\
\hline 250 & Verstoß gegen Verfahrensvorschriften \\
\hline 51 & Minderheitenschutz \\
\hline & Bekanntgabe der Entscheidung \\
\hline & Rechtsmittel \\
\hline
\end{tabular}

Christoph Kern
Christoph Kern
Christoph Kern
Christoph Kern
Christoph Kern
Christoph Kern
Christoph Kern
Christoph Kern
Christoph Kern

Dritter Abschnitt

Wirkungen des bestätigten Plans. Überwachung der Planerfüllung

$\$ 254 \quad$ Allgemeine Wirkungen des Plans

$\$ 254 \mathrm{a}$

$\$ 254 \mathrm{~b}$

$\$ 255$

$\$ 256$

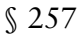

$\$ 258$

$\$ 259$

$\mathbb{} 259 \mathrm{a}$

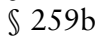

$\$ 260$

$\$ 261$

$\$ 262$

$\$ 263$

$\$ 264$

$\$ 265$

$\$ 266$

\$ 267

\268

$\$ 269$

Wirkung für alle Beteiligten

Wiederauflebensklausel

Vollstreckung aus dem Plan

Wirkungen der Aufhebung

Vollstreckungsschutz

Besondere Verjährungsfrist

Überwachung der Planerfüllung

Kreditrahmen

Nachrang von Neugläubigern

Berücksichtigung des Nachrangs

Aufhebung der Überwachung

Kosten der Überwachung
Rechte an Gegenständen. Sonstige Wirkungen des Plans

Streitige Forderungen. Ausfallforderungen

Aufhebung des Insolvenzverfahrens

Aufgaben und Befugnisse des Insolvenzverwalters

Anzeigepflicht des Insolvenzverwalters

Zustimmungsbedürftige Geschäfte

Bekanntmachung der Überwachung

\section{Siebter Teil}

Koordinierung der Verfahren von Schuldnern, die derselben Unternehmensgruppe angehören

Erster Abschnitt

Allgemeine Bestimmungen

Andreas Piekenbrock Andreas Piekenbrock Andreas Piekenbrock Andreas Piekenbrock Andreas Piekenbrock Andreas Piekenbrock Andreas Piekenbrock Andreas Piekenbrock Andreas Piekenbrock Andreas Piekenbrock Andreas Piekenbrock Andreas Piekenbrock Andreas Piekenbrock Andreas Piekenbrock Andreas Piekenbrock Andreas Piekenbrock Andreas Piekenbrock Andreas Piekenbrock Andreas Piekenbrock Andreas Piekenbrock

Andreas Piekenbrock Andreas Piekenbrock Andreas Piekenbrock 
Inhaltsübersicht

Zweiter Abschnitt

Koordinationsverfahren

$\$ 269 \mathrm{~d} \quad$ Koordinationsgericht

$\$ 269 \mathrm{e}$

$\$ 269 \mathrm{f}$

$\$ 269 \mathrm{~g}$

$\$ 269 \mathrm{~h}$

$\$ 269 \mathrm{i}$

Verfahrenskoordinator

Koordinationsplan

Andreas Piekenbrock

Aufgaben und Rechtsstellung des Verfahrenskoordinators

Vergütung des Verfahrenskoordinators

Abweichungen vom Koordinationsplan

Andreas Piekenbrock

Andreas Piekenbrock

Andreas Piekenbrock

Andreas Piekenbrock

Andreas Piekenbrock

Achter Teil

Eigenverwaltung

\$ 270

$\$ 270 \mathrm{a}$

\270b

$\$ 270 \mathrm{c}$

$\$ 270 \mathrm{~d}$

\ 271

\272

\ 273

\$2 274

\ 275

\276

\276a

\277

\278

\ 279

\280

\ 281

\282

$\mathbb{} 283$

$\int 284$

$\mathbb{} 285$
Voraussetzungen

Eröffnungsverfahren

Vorbereitung einer Sanierung

Bestellung des Sachwalters

Eigenverwaltung bei gruppenangehörigen Schuldner

Nachträgliche Anordnung

Aufhebung der Anordnung

Öffentliche Bekanntmachung

Rechtsstellung des Sachwalters

Mitwirkung des Sachwalters

Mitwirkung des Gläubigerausschusses

Mitwirkung der Überwachungsorgane

Anordnung der Zustimmungsbedürftigkeit

Mittel zur Lebensführung des Schuldners

Gegenseitige Verträge

Haftung. Insolvenzanfechtung

Unterrichtung der Gläubiger

Verwertung von Sicherungsgut

Befriedigung der Insolvenzgläubiger

Insolvenzplan

Masseunzulänglichkeit
Caroline Meller-Hannich Caroline Meller-Hannich Caroline Meller-Hannich Caroline Meller-Hannich Caroline Meller-Hannich Caroline Meller-Hannich Caroline Meller-Hannich Caroline Meller-Hannich Caroline Meller-Hannich Caroline Meller-Hannich Caroline Meller-Hannich Caroline Meller-Hannich Caroline Meller-Hannich Caroline Meller-Hannich Caroline Meller-Hannich Caroline Meller-Hannich Caroline Meller-Hannich Caroline Meller-Hannich Caroline Meller-Hannich Caroline Meller-Hannich Caroline Meller-Hannich 
